\title{
Equilibrium long-ranged charge correlations at the surface of a conductor coupled to the electromagnetic radiation II
}

\author{
Bernard Jancovic* * and Ladislav Šama用 \\ Laboratoire de Physique Théorique, Université de Paris-Sud \\ 91405 Orsay Cedex, France ${ }^{\ddagger}$
}

(Dated: December 3, 2018)

\begin{abstract}
Results of a previous article with the same title are retrieved by a different method. A onecomponent plasma is bounded by a plane surface. The plasma is fully coupled to the electromagnetic field, therefore the charge correlations are retarded. The quantum correlation function of the surface charge densities, at times different by $t$, at asymptotical large distances $R$, at inverse temperature $\beta$, decays as $-1 /\left(8 \pi^{2} \beta R^{3}\right)$, a surprisingly simple result: the decay is independent of Planck's constant $\hbar$ and of the time difference $t$. The present paper is based on the analysis of the collective vibration modes of the system.
\end{abstract}

PACS numbers: 05.30.-d, 52.40.Db, 73.20.Mf, 05.40.-a

\section{INTRODUCTION}

In a previous paper [1], we studied the asymptotic form of the two-point correlation function of the surface charge densities on a plane wall bounding a conductor [in the special case when the conductor is a one-component plasma (OCP), also called jellium], taking into account the retardation and the quantum nature of both the onecomponent plasma and the radiation. The novelty was the retardation; instead of assuming as an interaction the Coulomb potential only, in [1] the coupling was through the full electromagnetic radiation. In other words instead of assuming the velocity of light $c$ to be infinite, the full Maxwell equations were used.

The previous paper [1] used the elaborate formalism of Rytov [2], presented also in [3]. This formalism is macroscopic, using frequency-dependent dielectric functions. In the present paper, we retrieve the same results by a simpler method, based on the analysis of the collective vibration modes of the system. This method is partially microscopic. It has already been used in the non-retarded case [4], Sec. 4.

We use Gaussian units. The OCP is made of pointparticles of charge $e$, mass $m$, and number density $n$, immersed in a uniform neutralizing background of charge density $-n e$. We recall the geometry (Fig. 1). We use Cartesian coordinates; a point is $\mathbf{r}=(x, y, z)$. The OCP occupies the half-space $\Lambda_{1}=\{x>0\}$, the half-space $\Lambda_{2}=\{x<0\}$ is vacuum; the two half-spaces are separated by an plane wall, impenetrable to the jellium, at $x=0$. A point on the wall is $\mathbf{R}=(y, z)$.

After long calculations, a very simple result was found

\footnotetext{
¥Unité Mixte de Recherche No 8627-CNRS

*Electronic address: Bernard.Jancovici@th.u-psud.fr

${ }^{\dagger}$ On leave from Institute of Physics, Slovak Academy of Sciences, Bratislava; Electronic address: Ladislav.Samaj@savba.sk
}

in [1]:

$$
\begin{aligned}
\beta S(t, \mathbf{R}) & \equiv \beta \frac{1}{2}\langle\sigma(t, \mathbf{R}) \sigma(0, \mathbf{0})+\sigma(0, \mathbf{0}) \sigma(t, \mathbf{R})\rangle^{\mathrm{T}} \\
& \sim-\frac{1}{8 \pi^{2}} \frac{1}{R^{3}}, \quad R \rightarrow \infty
\end{aligned}
$$

where $\beta$ is the inverse temperature, $\langle\cdots\rangle^{\mathrm{T}}$ represents a truncated statistical average, $\sigma(t, \mathbf{R})$ is the surface charge density at time $t$ and at point $\mathbf{R}$ on the surface. This value (1) surprisingly is independent of $t$ and $\hbar$, boiling down to the classical result at time difference zero without retardation.

We define the Fourier transform of a function $f(\mathbf{R})$ as

$$
f(\mathbf{q})=\int d^{2} R \exp (i \mathbf{q} \cdot \mathbf{R}) f(\mathbf{R})
$$

A result equivalent to (1) is that the Fourier transform of its lhs, $\beta S(t, \mathbf{q})$, has a kink singularity at $\mathbf{q}=\mathbf{0}$, behaving at small $q$ like $q /(4 \pi)$.

The present paper is organized as follows. Sec. II is a general exposition of the formalism of vibration modes. Sec. III describes the contribution of surface modes (which are localized on both sides of the wall). Sec. IV describes the contribution of transverse modes delocal-

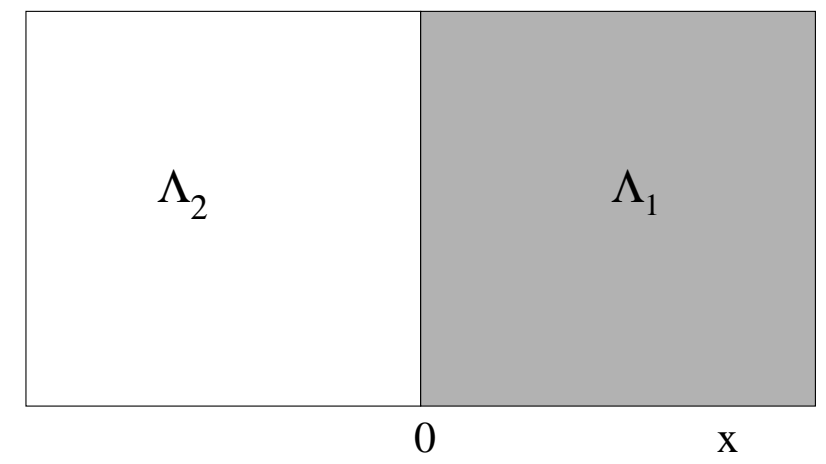

FIG. 1: The geometry. 
ized on the vacuum side. Sec. V describes the contribution of transverse modes delocalized on both sides. In Sec. VI, we recall the contribution of the longitudinal modes. Sec. VII is the Conclusion.

\section{COLLECTIVE VIBRATION MODES}

We consider a fluctuation of the surface charge density $\sigma$, of wavenumber $\mathbf{q}$ and frequency $\omega$, such that $\sigma$ is of the form

$$
\sigma(\mathbf{R}, t)=\sigma_{\mathbf{q} \omega}(t) \exp (i \mathbf{q} \cdot \mathbf{R})+\text { c.c. },
$$

where c.c. means complex conjugate. $\sigma_{\mathbf{q} \omega}(t)$ is a complex quantity, vibrating at frequency $\omega$. The emitted radiation, of frequency $\omega$, is described by the Maxwell equations (we use the microscopic ones, involving only the electric field $\mathbf{E}$ and the magnetic field $\mathbf{B}$, averaged in a suitable way [5]). The charge density is $\rho$, the electric current density is $\mathbf{J}$, the velocity of light is $c$. These Maxwell equations are

$$
\begin{aligned}
\nabla \times \mathbf{B} & =\frac{1}{c} \frac{\partial \mathbf{E}}{\partial t}+\frac{4 \pi}{c} \mathbf{J} \\
\nabla \times \mathbf{E} & =-\frac{1}{c} \frac{\partial \mathbf{B}}{\partial t} \\
\nabla \cdot \mathbf{E} & =4 \pi \rho \\
\nabla \cdot \mathbf{B} & =0
\end{aligned}
$$

In the quantum case, the quantities appearing in these equations are operators. In region $\Lambda_{2}, \rho=0$ and $\mathbf{J}=\mathbf{0}$, of course.

There are solutions to these Maxwell equations which are superpositions of transverse waves, which we shall study first. In region $\Lambda_{1}$, for these transverse waves, $\rho=$ 0. Furthermore, the Maxwell equations (44/7) have to be supplemented by

$$
\mathbf{E}=\frac{4 \pi}{\omega_{p}^{2}} \frac{\partial \mathbf{J}}{\partial t}
$$

where $\omega_{p}=\left(4 \pi n e^{2} / m\right)^{1 / 2}$ is the plasma frequency. This equation (88) is obtained from assuming that the OCP can be treated in a hydrodynamical linearized approximation where the velocity is $\mathbf{v}(\mathbf{r}, t)$ obeying the Newton equation $m \partial \mathbf{v} / \partial t=e \mathbf{E}$ (the term $e \mathbf{v} \times \mathbf{B}$ in the Lorentz force is suppressed by the linearization) and the current density is $\mathbf{J}=e n \mathbf{v}$ (the density is the constant $n$, again because of the linearization). It should be remarked that in the equation (8), there is no damping term; this absence of damping term is valid for the small wavenumbers which will be considered, and is a property special to the OCP.

Macroscopic Maxwell equations equivalent to (4-8) can also be obtained. Combining (4) and (8), at frequency $\omega$, we obtain

$$
\nabla \times \mathbf{B}=\frac{1}{c} \frac{\partial(\epsilon \mathbf{E})}{\partial t}
$$

where the frequency-dependent dielectric function is

$$
\epsilon(\omega)=1-\frac{\omega_{p}^{2}}{\omega^{2}}
$$

which is of the Drude form, without dissipation. Multiplying ([6), where $\rho=0$, by $\epsilon$ does not change anything if $\epsilon \neq 0$ (the case $\epsilon=0$ will be studied later). To an excellent approximation, the magnetic permeability is $\mu=1$. Thus we have obtained the macroscopic Maxwell equations [5], with now $\rho=0$ and $\mathbf{J}=0$.

For these transverse waves, the wavenumber vector has components $\left(k_{1}, \mathbf{q}\right)$ in region $\Lambda_{1}$ and $\left(k_{2}, \mathbf{q}\right)$ in region $\Lambda_{2}$, where $k_{1}$ and $k_{2}$ are the $x$ components (the wavenumbers have the same components parallel to the surface $\mathbf{q}=$ $\left(q_{y}, q_{z}\right)$ as a consequence of the boundary conditions, as will be shown later).

From the Maxwell equations (44.77) and (8), the dispersion relations are

$$
\omega^{2}=c^{2}\left(q^{2}+k_{2}^{2}\right)
$$

in region $\Lambda_{2}$, and

$$
\omega^{2}=\omega_{p}^{2}+c^{2}\left(q^{2}+k_{1}^{2}\right)
$$

in region $\Lambda_{1}$. Depending on the value of $\omega, k_{1}$ or $k_{2}$ are real or pure imaginary, as shown from (11) and (12). If $\omega^{2}<(c q)^{2}$, both $k_{1}$ and $k_{2}$ are pure imaginary. If $(c q)^{2}<\omega^{2}<\omega_{p}^{2}+(c q)^{2}, k_{1}$ is pure imaginary and $k_{2}$ is real. If $\omega^{2}>\omega_{p}^{2}+(c q)^{2}$, both $k_{1}$ and $k_{2}$ are real.

In addition to these plane transverse waves, in region $\Lambda_{1}$, when the frequency is $\omega_{p}$ (then $\epsilon=0$ ) the Maxwell equations have solutions which are longitudinal waves 5 , 6]. They have already been studied in [4].

The energy density in region $\Lambda_{2}$ is [5]

$$
U_{2}=\frac{1}{8 \pi}\left(E^{2}+B^{2}\right)
$$

the energy density in region $\Lambda_{1}$ is

$$
U_{1}=\frac{1}{8 \pi}\left(E^{2}+B^{2}\right)+\frac{2 \pi}{\omega_{p}^{2}} J^{2}
$$

A derivation of (14) is presented in the Appendix, as well as another form of it $[\underline{6}$.

At $x=0$, the existence of a surface charge density $\sigma$ is associated with a discontinuity of the $x$ component of the electrical field

$$
4 \pi \sigma=E_{x}^{+}-E_{x}^{-},
$$

where the upperscripts + or - mean approaching the plane $x=0$ from the regions $\Lambda_{1}$ or $\Lambda_{2}$, respectively. The other conditions at $x=0$ are the continuity of the components of the electric and magnetic fields parallel to the surface. From the conditions at $x=0$, one easily deduces that the $(y, z)$ components of the wavenumbers indeed are $\mathbf{q}$. Since the system is invariant by rotations around the $x$ axis, general results can be obtained by choosing $\mathbf{q}$ along the $y$ axis: $\mathbf{q}=\left(q_{y}, 0\right)$; then $q_{y}^{2}=q^{2}$. We consider only modes which contribute to $\sigma$. 


\section{III. $\omega^{2}<(c q)^{2}$ (POLARITONS)}

Since $k_{1}$ and $k_{2}$ are pure imaginary, the solutions of the Maxwell equations are of the form (the subscript $j=1,2$ denotes the region $\Lambda_{1}$ or $\Lambda_{2}$ ) [7]

$$
\begin{gathered}
\mathbf{E}_{j}=\left[a_{j}(t), b_{j}(t), 0\right] \exp \left(i q_{y} y-\kappa_{j}|x|\right)+\text { c.c. }, \\
\mathbf{B}_{j}=\left[0,0, d_{j}(t)\right] \exp \left(i q_{y} y-\kappa_{j}|x|\right)+\text { c.c. },
\end{gathered}
$$

where, from (11) and (12), $c \kappa_{2}=\sqrt{(c q)^{2}-\omega^{2}}$ and $c \kappa_{1}=\sqrt{\omega_{p}^{2}+(c q)^{2}-\omega^{2}}$. Since all fields are localized near the surface, these modes are called surface plasmons; polariton is also used when retardation, as here, is taken into account.

From the Maxwell equations and the conditions that $E_{y}$ and $B_{z}$ are continuous at $x=0$, in this Section there is a relation between $\omega$ and $\mathbf{q}$ :

$$
\omega^{2}=\omega_{p}^{2} / 2+(c q)^{2}-\sqrt{\left(\omega_{p}^{2} / 2\right)^{2}+(c q)^{4}} .
$$

Therefore, in this Section, $\sigma_{\mathbf{q} \omega}$ will be called $\sigma_{\mathbf{q}}$. $\omega$ has its non-retarded value $\omega_{p} / \sqrt{2}$ only at large $q$. For the small values of $q$, that we are interested in, $\omega$ behaves as $c q$. From (18) follows $\kappa_{1} \kappa_{2}=q^{2}$, a useful relation in the calculations (the detail of which is omitted) which follow.

Taking into account (15) and the continuity of $E_{y}$ and $B_{z}$, the Maxwell equations (5), (6) $[\rho=0$ in (6) $]$ give the prefactors $a_{i}, b_{i}, d_{i}$ as functions of $\sigma_{\mathbf{q}}$. One finds

$$
\begin{aligned}
& a_{1}(t)=\frac{\kappa_{2}}{\kappa_{1}+\kappa_{2}} 4 \pi \sigma_{\mathbf{q}}(t), \quad a_{2}(t)=-\frac{\kappa_{1}}{\kappa_{1}+\kappa_{2}} 4 \pi \sigma_{\mathbf{q}}(t) \\
& b_{1}(t)=b_{2}(t)=-i \frac{q_{y}}{\kappa_{1}+\kappa_{2}} 4 \pi \sigma_{\mathbf{q}}(t) \\
& d_{1}(t)=d_{2}(t)=\frac{i q_{y}}{c \kappa_{2}} \frac{1}{\kappa_{1}+\kappa_{2}} 4 \pi \dot{\sigma}_{\mathbf{q}}(t) .
\end{aligned}
$$

In region $\Lambda_{1}, \mathbf{J}$ can be obtained from (41). One finds

$$
\begin{aligned}
& J_{1 x}=-\exp \left(i q_{y} y-\kappa_{1} x\right) \dot{\sigma}_{\mathbf{q}}(t)+\text { c.c. }, \\
& J_{1 y}=\frac{i q_{y}}{\kappa_{2}} \exp \left(i q_{y} y-\kappa_{1} x\right) \dot{\sigma}_{\mathbf{q}}(t)+\text { c.c. }
\end{aligned}
$$

From (19) and (20), one computes the energy densities (13) and (14) and the total energy $H_{\mathbf{q}}$. Later, we shall consider that the large area $A$ of the wall goes to infinity; therefore, the oscillatory terms $\exp \left( \pm 2 i q_{y} y\right)$ do not contribute to the integral on $\mathbf{R}$. One finds

$$
\begin{aligned}
H_{\mathbf{q}} \equiv & \int_{A} d^{2} R\left[\int_{0}^{\infty} d x U_{1}+\int_{-\infty}^{0} d x U_{2}\right] \\
= & A 2 \pi\left(\frac{\kappa_{1}}{\kappa_{2}}+\frac{\kappa_{2}}{\kappa_{1}}\right) \frac{1}{\kappa_{1}+\kappa_{2}} \\
& \times\left[\left|\sigma_{\mathbf{q}}(t)\right|^{2}+\frac{1}{\omega^{2}}\left|\dot{\sigma}_{\mathbf{q}}(t)\right|^{2}\right] \\
\equiv & A C_{\mathbf{q}}\left[\left|\sigma_{\mathbf{q}}(t)\right|^{2}+\frac{1}{\omega^{2}}\left|\dot{\sigma}_{\mathbf{q}}(t)\right|^{2}\right]
\end{aligned}
$$

One sees that $H_{\mathbf{q}}$ is the energy of a two-dimensional harmonic oscillator (two-dimensional because $\sigma_{\mathbf{q}}(t)$ is a complex quantity). For such a quantum oscillator, where the variable $\sigma_{\mathbf{q}}(t)$ plays the role of the position variable, the contribution to $\beta S(t, \mathbf{q})$ is

$$
\beta S_{A}(t, \mathbf{q})=\frac{1}{C_{\mathbf{q}}} f(\omega) \cos (\omega t),
$$

where

$$
f(\omega)=\frac{\beta \hbar \omega}{2} \operatorname{coth} \frac{\beta \hbar \omega}{2} .
$$

Here, for $q \rightarrow 0, \omega \sim c q$, and (22) behaves like $q^{2}$. Therefore, the polaritons do not contribute to the retarded asymptotic form of $S(t, \mathbf{R})$.

On the contrary, in the non-retarded case $c \rightarrow \infty, \omega=$ $\omega_{s} \equiv \omega_{p} / \sqrt{2}$, and (22) becomes

$$
\beta S_{A}(t, q)=\frac{q}{2 \pi} f\left(\omega_{s}\right) \cos \left(\omega_{s} t\right)
$$

in agreement with [4].

$$
\text { IV. } \quad(c q)^{2}<\omega^{2}<\omega_{p}^{2}+(c q)^{2}
$$

Now $k_{1}$ is pure imaginary while $k_{2}$ is real. With $\mathbf{q}=$ $\left(q_{y}, 0\right)$, in region $\Lambda_{2}$ the general form of component $E_{x}$ must be

$$
E_{2 x}=\exp \left(i q_{y} y\right)\left[a(t) \cos \left(k_{2} x\right)+b(t) \sin \left(k_{2} x\right)\right]+\text { c.c. } .
$$

Using the Maxwell equations (5), (6) gives the other nonzero components of the fields in region $\Lambda_{2}$ as

$$
\begin{aligned}
& E_{2 y}=\exp \left(i q_{y} y\right) \frac{i k_{2}}{q_{y}}\left[-a(t) \sin \left(k_{2} x\right)+b(t) \cos \left(k_{2} x\right)\right]+\text { c.c. }, \\
& B_{2 z}=-\exp \left(i q_{y} y\right) \frac{1}{c q_{y}}\left[\dot{a}(t) \cos \left(k_{2} x\right)+\dot{b}(t) \sin \left(k_{2} x\right)\right]+\text { c.c. }
\end{aligned}
$$

In region $\Lambda_{1}$, since $k_{1}$ is pure imaginary, the fields depend on $x$ like $\exp \left(-\kappa_{1} x\right)$. The continuity of $E_{y}$ and $B_{z}$ at $x=0$ determine the coefficients in function of $a$ and $b$, and (3) and (15) give

$$
a(t)=\frac{\omega^{2}-\omega_{p}^{2}}{\omega_{p}^{2}} 4 \pi \sigma_{\mathbf{q} \omega}(t), \quad b(t)=-\frac{\kappa_{1} \omega^{2}}{k_{2} \omega_{p}^{2}} 4 \pi \sigma_{\mathbf{q} \omega}(t) .
$$

Let $\Lambda_{2}$ be the region $0>x>-L_{2}$ (with a large $L_{2}$, which at the end will be taken as infinite). Since $U_{1}$ has an exponential factor $\exp \left(-2 \kappa_{1} x\right)$, the region $\Lambda_{1}$ does not contribute to the total energy in this limit. The total energy only is $H_{\mathbf{q} \omega}=A \int_{-L_{2}}^{0} d x U_{2}$. Using (25,28) in (13) 
gives

$$
\begin{aligned}
& H_{\mathbf{q} \omega}=2 \pi A L_{2} \frac{\omega^{2}\left(\omega_{+}^{2}-\omega^{2}\right)\left(\omega^{2}-\omega_{-}^{2}\right)}{\omega_{p}^{2}(c q)^{2}\left[\omega^{2}-(c q)^{2}\right]} \\
& \times\left[\left|\sigma_{\mathbf{q} \omega}(t)\right|^{2}+\frac{1}{\omega^{2}}\left|\dot{\sigma}_{\mathbf{q} \omega}(t)\right|^{2}\right] \\
& \equiv A L_{2} C_{\mathbf{q} \omega}\left[\left|\sigma_{\mathbf{q} \omega}(t)\right|^{2}+\frac{1}{\omega^{2}}\left|\dot{\sigma}_{\mathbf{q} \omega}(t)\right|^{2}\right]
\end{aligned}
$$

where $\omega_{-}^{2}$ is given by (18) and $\omega_{+}^{2}$ is given by a modified (18) with a + sign in front of the square root. Therefore, the contribution of this mode to $\beta S(t, \mathbf{q})$ is

$$
\beta S_{B \omega}(t, \mathbf{q})=\frac{1}{L_{2} C_{\mathbf{q} \omega}} f(\omega) \cos (\omega t) .
$$

There are an infinity of modes of this kind, labeled by their frequency $\omega$, or, equivalently, by $k_{2}$. For obtaining the total contribution of these modes, $\beta S_{B}(t, \mathbf{q})$ to $\beta S(t, \mathbf{q})$, one has to take the sum on $k_{2}$ of (30). Since $L_{2}$ is large, $\sum_{k_{2}} \cdots=\left(L_{2} / \pi\right) \int d k_{2} \cdots$. Furthermore, $d k_{2}=d\left(\omega^{2}\right) /\left(2 c^{2} k_{2}\right)$. Therefore,

$$
\begin{aligned}
\beta S_{B}(t, \mathbf{q})= & \frac{1}{\pi} \int_{(c q)^{2}}^{\omega_{p}^{2}+(c q)^{2}} d\left(\omega^{2}\right) \frac{1}{2 c \sqrt{\omega^{2}-(c q)^{2}} C_{\mathbf{q} \omega}} \\
& \times f(\omega) \cos (\omega t) .
\end{aligned}
$$

After the change of variable $\omega^{2}=(c q)^{2}(1+u)$, (31) becomes, in the limit $q \rightarrow 0$,

$$
\beta S_{B}(t, \mathbf{q}) \sim \frac{q}{4 \pi^{2}} f(0) \cos 0 \int_{0}^{\infty} \frac{d u}{\sqrt{u}(1+u)}=\frac{q}{4 \pi} .
$$

(32) will be found the only contribution of order $q$ to $\beta S(t, \mathbf{q})$.

$$
\text { V. } \omega^{2}>\omega_{p}^{2}+(c q)^{2}
$$

Now, $k_{1}$ and $k_{2}$ are both real. The general form of the components $x$ of the electrical fields are

$$
E_{j x}=\exp \left(i q_{y} y\right)\left[a_{j}(t) \cos \left(k_{j} x\right)+b_{j}(t) \sin \left(k_{j} x\right)\right]+\text { c.c. } .
$$

(33) involves 4 coefficients, instead of 3 in $E_{i x}$ of Section IV (where $E_{1 x}$, not written explicitly, involves only 1 coefficient). Therefore the equations which were used in Section IV are not enough for determining all the coefficients in the fields as functions of $\sigma_{\mathbf{q} \omega}(t)$. Fortunately, parts of the fields are uncoupled to $\sigma$ and, for describing the modes coupled to $\sigma$, it is enough to choose in (33) $b_{i}=0$. Then, the Maxwell equations and the conditions at $x=0$ determine all the fields as functions of $\sigma_{\mathbf{q} \omega}(t)$. Assuming region $\Lambda_{i}$ to be of large length $L_{i}$ in $x$, one finds the total energy $H=H_{1 \mathbf{q} \omega}+H_{2 \mathbf{q} \omega}$ where the energy in region $\Lambda_{1}$ is

$$
\begin{aligned}
& H_{1 \mathbf{q} \omega} \\
& =A L_{1} \frac{2 \pi \omega^{2}}{\omega_{p}^{4}(c q)^{2}} \omega^{2}\left(\omega^{2}-\omega_{p}^{2}\right)\left[\left|\sigma_{\mathbf{q} \omega}(t)\right|^{2}+\frac{1}{\omega^{2}}\left|\dot{\sigma}_{\mathbf{q} \omega}(t)\right|^{2}\right] \\
& \equiv A L_{1} C_{1 \mathbf{q} \omega}\left[\left|\sigma_{\mathbf{q} \omega}(t)\right|^{2}+\frac{1}{\omega^{2}}\left|\dot{\sigma}_{\mathbf{q} \omega}(t)\right|^{2}\right]
\end{aligned}
$$

and in region $\Lambda_{2}$

$$
\begin{aligned}
& H_{2 \mathbf{q} \omega} \\
& =A L_{2} \frac{2 \pi \omega^{2}}{\omega_{p}^{4}(c q)^{2}}\left(\omega^{2}-\omega_{p}^{2}\right)^{2}\left[\left|\sigma_{\mathbf{q} \omega}(t)\right|^{2}+\frac{1}{\omega^{2}}\left|\dot{\sigma}_{\mathbf{q} \omega}(t)\right|^{2}\right] \\
& \equiv A L_{2} C_{2 \mathbf{q} \omega}\left[\left|\sigma_{\mathbf{q} \omega}(t)\right|^{2}+\frac{1}{\omega^{2}}\left|\dot{\sigma}_{\mathbf{q} \omega}(t)\right|^{2}\right] .
\end{aligned}
$$

For obtaining the total contribution of these modes, of different frequencies $\omega$, to $\beta S(t, \mathbf{q})$, it would be necessary to sum on $\omega$ the quantity

$$
\beta S_{C \omega}(t, \mathbf{q})=\frac{1}{L_{1} C_{1 \mathbf{q} \omega}+L_{2} C_{2 \mathbf{q} \omega}} f(\omega) \cos (\omega t) .
$$

We were not able to perform this sum. Fortunately, for $\omega$ close to $\omega_{p}$, (35) is negligible compared to (34) and it will turn out that the sum on $\omega$ involves only such values of $\omega$ in the limit of small $q$. Thus, we can neglect the term $L_{2} C_{2 \mathbf{q} \omega}$ in (36) writing

$$
\beta S_{C \omega}(t, \mathbf{q})=\frac{1}{L_{1} C_{1 \mathbf{q} \omega}} f(\omega) \cos (\omega t),
$$

and perform the sum on $\omega$ by replacing it by an integral like in Section IV. Therefore the contribution of these modes is

$$
\begin{aligned}
S_{C}(t, \mathbf{q})= & \frac{1}{\pi} \int_{\omega_{p}^{2}+(c q)^{2}}^{\infty} d\left(\omega^{2}\right) \\
& \times \frac{\omega_{p}^{4} f(\omega) \cos (\omega t)}{4 \pi c \sqrt{\omega^{2}-\omega_{p}^{2}-(c q)^{2}} \omega^{4}\left(\omega^{2}-\omega_{p}^{2}\right)}
\end{aligned}
$$

After the change of variable $\omega^{2}=\omega_{p}^{2}+(c q)^{2}(1+u)$, (38) becomes, in the limit $q \rightarrow 0$,

$$
\begin{aligned}
S_{C}(t, \mathbf{q}) & \sim \frac{q}{4 \pi^{2}} f\left(\omega_{p}\right) \cos \left(\omega_{p} t\right) \int_{0}^{\infty} \frac{d u}{\sqrt{u}(1+u)} \\
& =\frac{q}{4 \pi} f\left(\omega_{p}\right) \cos \left(\omega_{p} t\right) .
\end{aligned}
$$

\section{LONGITUDINAL MODES}

In addition to the transverse modes studied up to now, in region $\Lambda_{1}$, there are longitudinal modes of frequency $\omega_{p}$ (then $\left.\epsilon\left(\omega_{p}\right)=0\right)$ [5, 6]. They are solutions of the equations (44) with now $\rho \neq 0$ and $\mathbf{B}=0$; therefore they occur also in the non-retarded case already studied 
in [4]. These longitudinal modes are a superposition of waves with the electric field parallel to the wavenumber vector. Here, we summarize the calculations of [4], using a slightly different notation.

Since all modes have the same frequency but differ by the component $x$ of the wave vector, in (3) $\sigma_{\mathbf{q} \omega}(t)$ must be replaced by $\sigma_{\mathbf{q} k}(t)$. Since $\mathbf{B}=0$, (5) reduces to $\nabla \times \mathbf{E}=$ 0 , and $\mathbf{E}$ is derivable from a potential. This potential is of the form

$$
\phi=\left[a(t) \exp \left(i q_{y} y\right)+\text { c.c. }\right] \sin (k x)
$$

this form ensures that the $x$ component of the electric field

$$
E_{1 x}=-k\left[a(t) \exp \left(i q_{y} y\right)+\text { c.c. }\right] \cos (k x)
$$

is non-zero at $x=0$, thus is coupled by (15) to $\sigma_{\mathbf{q} k}(t)$ (in region $\Lambda_{2}$, there are no longitudinal waves, thus $\mathbf{E}_{2}=$ $0)$. (15) gives $-k a(t)=4 \pi \sigma_{\mathbf{q} k}(t)$. After $E_{2 y}$ has been expressed from (40), one finds for the energy

$$
H_{\mathbf{q} k}=4 \pi A L_{1} \frac{k^{2}+q^{2}}{k^{2}}\left[\left|\sigma_{\mathbf{q} k}(t)\right|^{2}+\frac{1}{\omega_{p}^{2}}\left|\dot{\sigma}_{\mathbf{q} k}(t)\right|^{2}\right] .
$$

After an integration on $k$, one finds for the contribution of the longitudinal modes

$$
\beta S_{D}(t, \mathbf{q})=\frac{1}{2 \pi^{2}} f\left(\omega_{p}\right) \cos \left(\omega_{p} t\right) \int_{0}^{K} d k \frac{k^{2}}{k^{2}+q^{2}},
$$

where $K$ is a cut-off beyond which the use of collective variables breaks down. For small $q$, the integral in (43) is $K-(\pi / 2) q+O\left(q^{2}\right)$; therefore, $\beta S_{D}(t, \mathbf{q})$ has a kink singularity at $\mathbf{q}=\mathbf{0}$ of the form

$$
\beta S_{D}(t, \mathbf{q}) \sim-\frac{q}{4 \pi} f\left(\omega_{p}\right) \cos \left(\omega_{p} t\right)
$$

\section{CONCLUSION}

(39) and (44) cancel each other. The only contribution of order $q$ to $\beta S(t, \mathbf{q})$ is (32). Thus

$$
\beta S(t, \mathbf{q}) \sim \frac{q}{4 \pi},
$$

the classical non-retarded result at $t=0$, as announced in the Introduction. This classical non-retarded result at $t=0$ was found previously as the result of two contributions: The one from the non-retarded surface plasmons $2 q /(4 \pi)$ and the one from the longitudinal bulk modes in the plasma $-q /(4 \pi)$. Things are different for the present retarded result, which comes from the fields in vacuum, with a cancellation of the contributions of the transverse waves and the longitudinal waves in the plasma.

An open qualitative problem is to understand by a physical argument why the retarded quantum result at an arbitrary $t$ is so simple, not involving $\hbar$ nor $t$.

\section{Acknowledgments}

L. Šamaj is grateful to LPT for its very kind hospitality. The support received from the European Science Foundation (ESF "Methods of Integrable Systems, Geometry, Applied Mathematics") and from the Grant VEGA 2/6071/2008 is acknowledged.

\section{APPENDIX}

The energy density of a one-component plasma (14) can be obtained from the Maxwell equations (447) and the force equation (8). Energy conservation can be expressed as [5]

$$
\frac{1}{8 \pi} \frac{\partial}{\partial t}\left(E^{2}+B^{2}\right)+\mathbf{J} \cdot \mathbf{E}=-\nabla \cdot \mathbf{S},
$$

where

$$
\mathbf{S}=\frac{c}{4 \pi}(\mathbf{E} \times \mathbf{B})
$$

is the Poynting vector (energy flow) and $\mathbf{J} \cdot \mathbf{E}$ is the rate per unit volume of doing work by the electric field. (8) gives

$$
\mathbf{J} \cdot \mathbf{E}=\frac{2 \pi}{\omega_{p}^{2}} \frac{\partial\left(J^{2}\right)}{\partial t}
$$

The lhs of (A.1) is the time derivative of the energy density, thus which is (14).

The last term of (14) can be expressed in terms of $\mathbf{E}$. One has to be careful about the sign: since oscillatory terms are to be discarded, $J^{2}=|\mathbf{J}|^{2}$ and $E^{2}=|\mathbf{E}|^{2}$. Using again (8), at frequency $\omega$, one can replace the last term in (14) by $\omega_{p}^{2} E^{2} /\left(8 \pi \omega^{2}\right)$. Taking into account (10), we obtain

$$
U_{1}=\frac{1}{8 \pi}\left[\frac{d(\omega \epsilon)}{d \omega} E^{2}+B^{2}\right],
$$

as written in [6].
[1] L. Šamaj and B. Jancovici, Equilibrium long-ranged charge correlations at the surface of a conductor coupled to the electromagnetic radiation, to appear in Phys. Rev. E.
[2] S. Rytov, Sov. Phys. JETP 6, 130 (1958).

[3] E. M. Lifshitz and L. P. Pitaevskii, Statistical Physics, Part 2, Chapter VIII (Pergamon Press, Oxford, 1980).

[4] B. Jancovici, J. Stat. Phys. 39, 427 (1985). 
[5] J. D. Jackson, Classical Electrodynamics, 2nd ed. (Wiley, New York, 1975).

[6] L. Landau and E. Lifshitz, Electrodynamics of Continuous Media (Pergamon Press, Oxford, 1960).
[7] J. M. Pitarke, V. M. Silkin, E. V. Chulkov, and P. M. Echenique, Rep. Prog. Phys. 70, 1 (2007). 